\title{
Los discursos sobre la modernización de los Servicios Públicos de Empleo: ¿hacia una nueva forma de gobernanza?
}

\author{
Carlos Jesús FERNÁNDEZ RODRÍGUEZ \\ Universidad Autónoma de Madrid \\ carlos.fernandez@uam.es \\ María Paz MARTín MARTín \\ Universidad Complutense de Madrid \\ paz.martin@egeco.es
}

Recibido: 08-05-2013

Aceptado con modificaciones: 16-12-2013

Aceptado finalmente: 20-01-2014

\begin{abstract}
Resumen
Las transformaciones en el modelo organizativo e institucional de la administración pública, que se vienen produciendo en gran número de estados de bienestar europeos desde los años ochenta, han sido una de las respuestas más consensuadas para hacer frente a una supuesta crisis de la cuestión social en el seno de la Unión Europea. Esta reforma administrativa, apoyada sobre herramientas de definición teórica como la nueva gestión pública, la gestión por objetivos o el partenariado, entraña transformaciones semánticas ("reparto de responsabilidades", "coste efectivo" o la sustitución de la noción de ciudadano administrado por la de cliente) y políticas (predominio de la dispersión del poder e individualización de las responsabilidades) que operan en los marcos de actuación y relación cívica entre el Estado y el individuo. Los paradigmas de la activación y la flexiguridad albergan este proyecto de modernización de la administración. Esta apuesta por una nueva gobernanza de lo social tendrá importantes consecuencias para los fundamentos políticos y morales de la cohesión social. En el sistema de empleo español, como en otros de Europa, esta reforma se ha planteado en clave de "modernización" del servicio público de empleo. El presente trabajo pretende ahondar en las representaciones de "modernización" que se desprenden de los debates en torno a la reestructuración de los servicios de empleo, haciendo previamente alusión a la trayectoria que ha seguido el proceso de reforma administrativa de los servicios públicos de empleo desde su despegue en la década de los ochenta hasta el inicio de la crisis.
\end{abstract}

Palabras clave servicio de empleo, políticas activas, modernización, partenariado, flexiguridad, gobernanza 


\title{
Discourses on Modernization in the Employment Public Services: Towards a New Form of Governance?
}

\begin{abstract}
The "crisis of the social issue" in the EU has led to a certain consensus in the need to renew the organizational and institutional model of public administration. The core of the reform implies important administrative changes in most of the European welfare states. Those changes are inspired on theories such as the new public management, management by objectives or partnership. Such changes involve both semantic ("sharing responsibilities", "effective costs", or the substitution of "citizen under an administration" by "consumer") and political (predominance of scattered forms of power and the individualization of responsibilities) transformations which operate in the framework of individuals and State relations. The paradigms of activation and flexicurity have been central in this public administration modernization project. This commitment with new forms of governance of social issues has important consequences for the political and moral foundations of social cohesion, and the Spanish case is not an exception. This paper aims at looking at those representations of "modernization" (as they appear in debates about the employment services restructuring policies) in detail as well as providing references to the trajectory of such reforms of public services since the early eighties to the beginning of the crisis.
\end{abstract}

Key words: employment services, active policies, modernization, partnership, flexicurity, governance.

\section{Referencia normalizada}

Hernández-Carr, A. (2014). "El salto a la nueva extrema derecha: una aproximación a los votantes de Plataforma per Catalunya”. Política y Sociedad, Vol.51 Núm.1 177-200

Sumario: Introducción. 1.Flexiguridad y modernización de los servicios públicos de empleo en España. 2.El discurso de los actores sociales en torno a la modernización de los servicios de empleo. 3.Conclusiones. "Nos coordinamos entre nosotros": poder y responsabilidad en un contexto de cambio. Bibliografía. 


\section{Introducción: el advenimiento de nuevos modelos de gobernanza.}

A lo largo de la última década, un conjunto de investigaciones académicas han hecho hincapié en las transformaciones que han sufrido las políticas sociales de empleo en la mayoría de los países occidentales a lo largo de las últimas dos décadas (por ejemplo, Sol y Westerweld, 2005; Serrano Pascual y Magnusson, 2007; Kallenberg, 2009; Landa Zapirain, 2009; Miguélez y Prieto, 2009). La Unión Europea ha seguido el hilo de estas transformaciones poniendo énfasis, en la actualidad, en un enfoque activo e individualizado del problema del desempleo. Esta nueva orientación ha permitido la consolidación de nuevos paradigmas en las políticas sociales de empleo actuales (a nivel tanto europeo como nacional) como son los de la activación y la flexiguridad, que se han convertido en eje de las estrategias europeas relacionadas con el empleo (Martín Artiles, 2002; Wilthagen y Tros, 2004; Keune \& Jepsen, 2007; Serrano Pascual, 2009a). A partir de 2007, el concepto de flexiguridad recoge este otro de activación para hacerlo formar parte de una propuesta global de regulación del mercado de trabajo donde el significado de seguridad en el empleo y en desempleo se transforma en la dirección de la seguridad activadora o el auto-aseguramiento (Martín Martín, 2009 y 2013). De modo que, los paradigmas de la activación y la flexiguridad no sólo no han entrado en crisis durante estos últimos años, sino que se han reforzado.

La hegemonía actual de estos paradigmas no puede ser entendida sin tener en cuenta otros procesos de reforma administrativa que llevan desarrollándose en el mundo occidental desde hace más de tres décadas, como reacción a una situación de crisis del Estado del Bienestar, y cuya raíz común es la necesidad de dar respuestas a retos derivados de un escenario más complejo que aquel en el que actuaba el clásico Estado-Nación clásico. Si en el período keynesiano las políticas sociales se planteaban desde un marco institucional y burocrático nacional, en el que la negociación de actores sociales con intereses contrapuestos jugaba un papel fundamental en la definición de las mismas, el contexto actual nos remite a un entorno caracterizado por la globalización económica, una nueva economía del conocimiento basada en las nuevas tecnologías y la producción flexible, la emergencia de una sociedad de redes, la crisis fiscal del Estado y una fragmentación de las identidades colectivas (Alonso, 2007). Este aumento de la complejidad y el crecimiento de las relaciones interdependientes entre distintos actores sociales (con la consiguiente dispersión de poderes) ha favorecido la emergencia de una nueva forma de entender la administración: la denominada gobernanza. En nombre de la gobernanza se articulan nuevas vías de intervención de la administración pública en la gestión de sus administrados (Pollitt, 1993), definidas como "modernas" y "eficaces" en 
contraposición a la gestión pública tradicional, tachada de ineficaz y excesivamente burocrática (Peters, 2005; Du Gay, 2012).

En este trabajo nos preguntamos por el carácter que el sentido de la reforma y la modernización adquiere en el contexto español de los servicios de empleo a la luz de los paradigmas hegemónicos de la activación y la flexiguridad. Para ello, indagamos en las transformaciones semánticas y políticas que traslucen los discursos de algunos de los actores políticos y sociales más representativos en el ámbito del empleo al respecto de dicha reforma o modernización (reestructuración de los servicios de empleo y sus modos de intervención), con el fin de conocer cómo interpretan la reforma y modernización del servicio de empleo los actores implicados en el mismo. El objetivo es el de describir estos discursos, representativos, de alguna manera, del imaginario social contemporáneo (Alonso y Fernández Rodríguez, 2013). Este análisis se complementa con una referencia previa a la trayectoria que ha seguido el proceso de reforma administrativa de los servicios públicos de empleo desde su despegue en la década de los ochenta hasta el comienzo de la crisis económica. Hemos optado por detener en ese momento nuestro análisis una vez que todo parece indicar que se está generando un escenario novedoso en lo concerniente a la articulación de la relación capital-trabajo a nivel de la UE (ver Alonso y Fernández Rodríguez, 2012; Laval y Dardot, 2013), cuyas consecuencias deben ser todavía valoradas con detenimiento en el caso español, una vez que estamos asistiendo a numerosos cambios, en buena medida, derivados de la profusión de reformas laborales desde la primavera de 2010 y que pueden tener una influencia en las percepciones sociales actualmente existentes en torno a las políticas de la UE y su imagen entre la ciudadanía.

El material empírico para este trabajo lo constituyen los datos cualitativos extraídos de un conjunto de entrevistas realizadas a diferentes actores sociales que participan o pretenden participar en la puesta en práctica de políticas activas de empleo, durante un período que se extendió desde octubre de 2008 hasta mayo de 2009. Las entrevistas se realizaron en el contexto de dos estudios de caso en las CCAA de Andalucía y Comunidad Valenciana; sobre los textos obtenidos se han aplicado técnicas propias de un análisis crítico del discurso (siguiendo a Fairclough, 1995; Wodak y Meyer, 2009) ${ }^{1}$. Así, la presente contribución se estructura en tres

${ }^{1}$ En esta investigación, se realizaron diecinueve entrevistas a diversos interlocutores sociales, entre ellos miembros de los sindicatos UGT y CCOO (a niveles tanto regional como nacional); representantes de la FEMP (Federación Española de Municipios y Provincias, también a nivel nacional y regional de las dos CCAA analizadas, Andalucía y Comunidad Valenciana); diversos trabajadores y gestores de políticas activas del INEM (Instituto Nacional de Empleo) y de los Servicios Andaluz y Valenciano de Empleo (SAE y SERVEF, respectivamente); y, finalmente, representantes tanto de la ONG Cáritas como de la Asociación de Grandes Empresas de Trabajo Temporal (AGETT), ambos a nivel nacional. Se incluye esta información en un anexo al final del artículo. Metodológicamente, las 
secciones: la primera pone de manifiesto la relación entre las prescripciones del paradigma de la flexiguridad y sus orientaciones a favor de la activación y los postulados de la nueva gestión pública, ofreciendo posteriormente una aproximación al contexto español de reformas administrativas en el ámbito del empleo; la segunda se dedicará al análisis de los discursos de los diferentes actores que participan en la puesta en marcha de estas políticas con el fin de alcanzar una mayor comprensión del proceso/propuesta de "modernización", tal y como se construye políticamente desde las distintas perspectivas; la tercera concluirá con una discusión sobre los principales resultados de nuestra investigación.

\section{Flexiguridad y modernización de los servicios públicos de empleo en España.}

El conocido concepto (y estrategia política) de la flexiguridad, que trata de combinar armónicamente dos nociones contrapuestas tradicionalmente como flexibilidad laboral y seguridad, constituye una "categoría resumen" de un nuevo marco interpretativo y de intervención en relación al tipo de (des)equilibrio que ha de darse entre protección social/justicia social y desarrollo económico. Este concepto trata de dar entidad a un modelo de gestión en el que en complementación convivan las políticas activas (incluidas, la intervención individualizada y las políticas específicas) y un tipo de gestión basada en el "reparto de responsabilidades, colaboración, cooperación, negociación (bargaining), diálogo social, partenariado (partnership) entre actores sociales y niveles de gobierno” y reducción del gasto público (hacer los “costes efectivos”), en la línea de la Nueva Gestión Pública (Martín Martín, 2009). La flexiguridad contiene no sólo una descripción de los problemas y las soluciones sino que también integra una definición de los instrumentos y procesos adecuados para alcanzar tales soluciones. Integrado en este paradigma, la activación será uno de los ejes fundamentales de intervención, poniendo énfasis en la necesidad de intervenir en las competencias, motivaciones y actitudes individuales de los desempleados, entendidos como sujetos individuales.

La flexiguridad ha sido considerada como parte esencial de los nuevos modos de gestión y gobernanza (instrumentos, procesos y actores) que, a día de hoy, aparecen como referente de actuación para los distintos Estados de Bienestar europeos (Bonvin, 2004; Serrano Pascual, 2009a y 2009b) copando el terreno mismo de la propuesta de activación que constituye uno de los vértices del llamado "triángulo de oro de la flexiguridad" y dos (si no tres) de los cuatro pilares de la propuesta europea de flexiguridad misma (Tovar y Revilla, 2012; Martín Martín, 2009 y

entrevistas tuvieron una duración de una hora y contaban con un guión previo organizado en torno a cuestiones relacionadas con la gestión del servicio público de empleo. El desarrollo de las entrevistas ha seguido las pautas establecidas en trabajos clásicos como los de Silverman (1993), Kvale (1996) o Vallés (2002). 
2013). Se ha identificado, por tanto, con una "modernización", en el sentido de evolución y mejora de los servicios. En las últimas décadas, la modernización se ha asociado a la puesta en práctica de los preceptos de la denominada Nueva Gestión Pública (New Public Management), cuyos principios afectan a tres aspectos constitutivos de la intervención administrativa, de manera complementaria: la financiación, el proceso de implementación y los actores que participan en dicha implementación. En términos de financiación, se tiende a adoptar una estrategia de gobierno favorable al recorte o contención del gasto social, adherida a la lógica "coste-beneficio". El proceso de implementación, antes ligado a los principios de orden, planificación y jerarquía propios del gerencialismo burocrático, va a ser desplazado por una nueva "gestión por objetivos" (MBO, Management By Objectives) en la que se enfatizan criterios propios de la gestión empresarial: entre ellos, los de eficiencia, evaluación y consecución de objetivos, con unos principios de actuación orientados en efectos y resultados; preferencia por estructuras organizacionales horizontales y autónomas, cuyas relaciones se rigen por contratos o cuasi-contratos; la multiplicación de procesos contractuales, de externalización y asociación; y un enfoque más individualista (Pollitt, 2003: 27-28). Finalmente, se produce una incorporación de nuevos actores a la implementación y gestión de las políticas sociales (empresas privadas, asociaciones, organizaciones no gubernamentales, sindicatos, etc.), que proveen servicios mediante contratos, acuerdos o negociaciones con las autoridades públicas, y que están sujetos a una auditoría de sus actividades.

Se configura, así, una estructura triangular en la provisión del bienestar, donde intervienen tres figuras relacionadas entre sí contractualmente: una parte principal (el Estado u otro organismo público), el agente contratado (ente privado u administración autonómica) y el cliente (el ciudadano). Esta dinámica contractual tiene, como efecto más destacado, una cierta difuminación de la labor del Estado como principal garante del bienestar social y, consecuentemente, plantea un importante riesgo de desmantelamiento de los marcos de responsabilidad colectiva, al surgir nuevos espacios simbólicos de negociación de las posiciones de los actores. Por otra parte, alimentando esta tendencia, la intervención más personalizada y adaptada a cada individuo abre la posibilidad de que una nueva versatilidad se instale en la relación entre implementador de la práctica política y el ciudadano, ya que deja de existir una norma rígida de intervención. Expresiones como las de "reparto de responsabilidades" o "gobierno a múltiples niveles" van a hacer referencia a una nueva tendencia de "gestión difusa", suponiendo la dilución de un referente público y la reinvención del rol del Estado.

Para entender el desarrollo de las reestructuraciones institucionales y nuevas lógicas de gobierno en el ámbito del empleo español, es preciso remitirse a los factores que están en la base misma de la instalación de un sistema de bienestar tras el franquismo. La institucionalización de dicho sistema, minimalista y tardía, tuvo un reflejo en las diversas medidas legislativas aprobadas en el período democrático en relación a las políticas de empleo, más orientadas a garantizar la convergencia económica con Europa que la social (Navarro, 2006). Así, en la primera etapa de la 
democracia se mantiene una óptica keynesiana en lo que se refiere a la regulación del mercado de trabajo, en un contexto de graves problemas derivados de la crisis económica que había desnudado las debilidades del capitalismo tardío español (Koch, 2006; Alonso, 2007). La Ley Básica de Empleo (51/1980) establece la creación del Instituto Nacional de Empleo (INEM), como parte de la gestión estatal de la política de empleo. El INEM supone un modelo de intervención monolítica, al reunir en sí la gestión de las prestaciones por desempleo y los servicios de colocación y gestión de las subvenciones de contratación y la formación no reglada. Sin embargo, esta ley incluye además un tímido gesto de superación de la rigidez: se abre la posibilidad de que el INEM colabore con instituciones y entidades especializadas para la orientación, formación y empleo de grupos con especiales dificultades de colocación (artículo 14.2), y se contempla el establecimiento de conciertos con empresas privadas y públicas orientados a la colocación de trabajadores en desempleo (artículo 15).

Desde mediados de los ochenta hasta nuestros días, con los gobiernos del PSOE y del PP, se observa una tendencia hacia la flexibilización de la regulación laboral en la mayoría de sus dimensiones, con paulatinas restricciones en el acceso a la prestación por desempleo y un reforzamiento del impulso de las políticas activas, a través de varias reformas de la legislación laboral. Asimismo, una progresiva descentralización del servicio público de empleo hacia las CCAA tendrá lugar como resultado del traspaso de políticas activas, programas de autoempleo, ayudas a "minusválidos"² (asistencia) y formación profesional ocupacional. A este proceso se suman las modificaciones del sistema de empleo introducidas por la importante Ley 10/1994: legalización de las agencias de colocación privadas sin fines lucrativos y debidamente autorizadas; creación del sistema integral para el empleo (SIPE, regulado en el RD. 75/1995); legalización de las Empresas de Trabajo Temporal (las conocidas ETT), y consagración de la contratación directa por el empresario. Este proceso ha provocado que algunos autores hayan dado por sentada la sustitución de un modelo intervencionista (en el espíritu de la Ley 1943, que regía el sistema de relaciones laborales franquistas) por uno liberal, tendente a la mínima regulación pública ${ }^{3}$. Sin embargo, estos cambios no proceden sólo de la conciencia de su conveniencia por parte de los actores políticos e interlocutores sociales, sino que se han visto favorecidos, alentados y condicionados por la influencia de instituciones internacionales, como las recomendaciones y directrices de la Unión Europea (por ejemplo, European Comission, 2007) o los Convenios de la OIT.

\footnotetext{
2 Mantenemos las denominaciones al uso en este momento, queriendo referir aquí "personas con diversidad funcional".

${ }^{3}$ Es importante señalar que otros autores consideran que el sistema nunca fue intervencionista, pues al estar demasiado burocratizado y contar con grandes vacíos legales, su capacidad de acción era más bien mecánica dejando un amplio margen de actuación a las empresas de selección privadas (Sobrino González, 2007).
} 
El proceso de descentralización territorial (concluido en el año 2000, aunque quedando fuera del mismo País Vasco, Ceuta y Melilla) se materializa jurídicamente con la Ley de Empleo 56/2003. En esta, se establece y reconoce el nuevo Sistema Nacional de Empleo, en el que se integran un re-denominado Servicio Público de Empleo Estatal y los distintos Servicios Públicos de Empleo Autonómicos (LO 9/1992). A ellos van a ser cedidas las funciones del anterior INEM, bajo el amparo de una legislación básica estatal: así, se ocuparán de la gestión de las prestaciones por desempleo; intermediación; gestión de las subvenciones a la contratación; formación profesional no reglada; delineación de la estrategia en política de empleo más adecuada a las necesidades de la Comunidad, y realización de Convenios con agencias de colocación, así como prácticas de colaboración con entidades asociadas. Además, en esta ley se delimitan los agentes que pueden participar en la intermediación laboral: Servicios Públicos de Empleo, agencias de colocación no lucrativas, ETT y "entidades colaboradoras de los servicios públicos de empleo” (Pérez Domínguez, 2007).

El proceso modernizador de los servicios públicos de empleo en España ha seguido, por lo tanto, dos vías paralelas: por un lado, la descentralización autonómica, que dará lugar a cuasi-contratos internos entre distintos niveles administrativos; por otro, la que atiende al reconocimiento legal y regulación de otros agentes de carácter privado, y que se consagra en la Ley 10/1994 (abriendo el régimen legal de contratos entre agentes privados y AAPP, según procedimientos de autorización y concurso). No obstante, tras completarse las transferencias, se generará una verdadera "cascada contractual", pues las CCAA se convertirán en potencial "parte principal" de los contratos establecidos con proveedores (corporaciones locales y entidades privadas), al tiempo que serán "contraparte" con respecto a la "parte principal” estatal, con la que se iniciará una dinámica de suscripción de acuerdos de colaboración. Algunos autores han considerado que el funcionamiento de este entramado está lejos de representar algo que pueda calificarse de "gerencialismo", estando más cercano a un modelo burocrático tradicional en completa desorganización (Valdés Dal-Ré, 2006) que debe enfrentarse a problemas crónicos de elevado desempleo, sobre todo en la actualidad (Santos Ortega y Martín Martín, 2012).

Tenemos, así, un modelo de gestión de las políticas de empleo caracterizado por una enorme complejidad contractual, pretensiones de adaptación a los supuestos de la Nueva Gestión Pública y, por tanto, la participación de actores diversos que juegan un papel importante en la gestión de estos servicios. En la siguiente sección, analizando los discursos de estos actores, pretendemos desvelar los sentidos de esta reforma y modernización de la gestión de las políticas de empleo en España a finales de los años 2000, un momento de crecimiento espectacular del desempleo que, sin embargo, no parecía anticipar el escenario de austeridad y reformas en el que nos encontramos en el momento de redactar este artículo. 


\section{El discurso de los actores sociales en torno a la modernización de los servicios de empleo.}

Una primera aproximación al discurso de los actores sociales parece mostrar la existencia de un acuerdo, prácticamente unánime, a la hora de diagnosticar la situación de los servicios públicos de empleo: todos parecen coincidir en que no funcionan. Los actores entrevistados manifiestan un aparente consenso en señalar los problemas del modelo clásico de intervención centralizada (cuya toma de decisiones se organiza jerárquicamente, siguiendo una estructura top/down), donde la gestión se llevaría directa y exclusivamente por parte de las administraciones públicas, y en donde los poderes centrales asumen la responsabilidad de diseñar políticas uniformes, inspiradas bajo un principio de igualdad de oportunidades y administradas burocráticamente. El análisis de los discursos revela que la modernización, para los entrevistados, estaría representada por la puesta en práctica de un modelo más dinámico, que podríamos definir como de gobernanza $a$ múltiples niveles, en donde se reclama la voz y la participación de multitud de actores en diversas etapas del proceso de intervención (diseño, implementación, evaluación), y que es con matices el vigente en el período del trabajo de campo. A través de este modelo, los actores reivindican su participación y una mayor coordinación, aunque a la vez se objeta su carácter inconcluso y los obstáculos para una gestión de calidad que plantean las deficiencias existentes en su diseño. Sin embargo, y a pesar de este consenso que comulga con el discurso hegemónico de la modernización, existen algunos matices diferenciadores importantes entre actores, según su naturaleza, más social (sindicatos, ONGs) o más económica (ETTs) así como ambivalencias y contradicciones en el discurso de un mismo actor. Así lo comprobamos a través de los distintos ejes de análisis y ponemos de manifiesto en la sección conclusiva. Este nuevo modelo de gobernanza, basado en la lógica del contrato y del incentivo, es una herramienta que sirve a la implementación de políticas de activación, igualmente fundadas sobre la lógica del contrato -con el usuario, como forma de responsabilizarlo de su situación- y la lógica del incentivo al trabajo, a través de una intervención personalizada, disciplinante y socializante-. Los recursos semánticos sobre los que se construyen estos discursos que justifican y proyectan la modernización serán analizados a continuación.

Hemos distinguido tres líneas discursivas (los antes mencionados "ejes de análisis"): la crítica al modelo burocrático anterior (1), la definición del nuevo modelo basado en la participación "equilibrada" de todos los actores, según dinámicas de partenariado y coordinación (2), y el carácter (los contenidos y los objetivos) de la intervención en materia de desempleo (la atención individualizada) que se pretende imprimir a las políticas a través de estos métodos descentralizados y contractuales (3). Tratamos así de deconstruir los términos en torno a los cuales se problematizan algunas cuestiones y se naturalizan otras, y donde, a un mismo tiempo, se expresan las interpretaciones hegemónicas acerca del funcionamiento de lo que deben ser las instituciones modernas, revisando las propuestas más discutidas sobre el aclamado espíritu de la modernización. 


\subsection{De la burocracia a la eficacia.}

Los problemas del actual modelo de intervención se plantean, en los discursos de los actores, como problemas heredados de una cierta forma de intervención, no adaptada a los nuevos tiempos. En este sentido, en las entrevistas se hicieron referencias al desfase del modelo frente a la realidad actual, cambiante, que exigiría una intervención mucho más ágil y flexible, ya que el marco de las políticas activas de empleo está "muy desfasado con respecto a las necesidades de la gente", tal como afirma un responsable Técnico de la FEMP a nivel nacional. El Servicio Público de Empleo es, así, criticado por su falta de eficacia, que deriva de varios problemas: falta de recursos humanos y dotaciones; centralización de sus actividades y consiguiente rigidez; la falta de cercanía con la realidad del desempleo que repercute en una intervención insuficiente o inadecuada; exceso de burocracia. Tratando de contrarrestar estas deficiencias, los discursos de los actores insisten en que la intervención en una realidad social marcada por cambios veloces y constantes requeriría de otras virtudes, como la adaptabilidad o la flexibilidad.

Se observa en estos discursos una preferencia por intervenciones más descentralizadas, que tengan en cuenta la dimensión local (más cercana), y por la especialización técnica. De lo contrario, se generará esa rigidez que impide dar soluciones a los problemas reales de los desempleados. La intervención burocratizada es considerada como una estructura que impone modelos homogéneos a situaciones diversas, y que conlleva anquilosamiento y genera lentitud, encorsetamiento, falta de reacción ante los problemas y falta de calidad en el servicio, dentro de un discurso anti-burocrático similar al de los gurús empresariales (Alonso y Fernández Rodríguez, 2006; Fernández Rodríguez, 2007). En este sentido, las ETT encuentran su oportunidad para desarrollar un discurso legitimador de su intervención. Desde ellas, se redunda en apuntar una falta de calidad de lo público; frente a ello ofrecen las bondades de los servicios facilitados desde el mercado, por parte de empresas expertas. Su discurso deslegitimador de lo público se apoya sobre argumentaciones de carácter técnico más que ideológico, como que "no pueden” o "no tienen”. Un ejemplo sería el siguiente:

"Claro, y si no los buscamos, sobre todo ahora, antes nos demandaban, ahora hay que salir a buscarlos, hay que ponerse las zapatillas y recorrer, vamos Polígono Industrial tras Polígono industrial, cosa que el servicio público de empleo no hace, no porque no quieran sino porque están completamente bloqueados de todo tipo de tareas (...) y están haciendo una excelente labor pero es que no pueden, no tienen medios para hacerlo, o sea, es que necesitas ... (...) con la descentralización de las políticas activas, nosotros tenemos una unidad de mercado que los servicios públicos de empleo no tienen (...)" (representante AGETT).

El discurso de las ETT (apoyado implícitamente por los responsables de los servicios de empleo) nos conduce hacia un nuevo paradigma donde la inflexibilidad, la centralización y la mala calidad de lo público se oponen a otro discurso, el de la 
calidad del servicio. Éste defiende una nueva manera de gestionar y nuevas formas de gobernanza basadas en las bondades del mercado frente a los fallos del Estado. En la misma línea de proporcionar un servicio de calidad se sitúa el discurso de los servicios públicos de empleo. Éstos parecen defender la provisión de servicios "cercanos" y "adecuados" a cualquier precio de modo que los ciudadanos dentro de este esquema se presentan como meros consumidores de los mismos.

"Nuestro principio es acercar al ciudadano los servicios ofreciéndole todos los servicios y dar el servicio más adecuado". (Jefe de servicio de gestión de la intermediación laboral SERVEF).

Por su parte, los actores sindicales, si bien están de acuerdo acerca de este mal funcionamiento de lo público, manifiestan un disenso respecto a los motivos de esta falta de calidad. Para ellos lo público no es esencialmente incompetente en la provisión de servicios, sino que se encuentra aquejado de problemas concretos y solucionables, como son la falta de financiación y la falta de personal. Para los sindicatos, la modernización sólo puede realizarse desde el ámbito público, cerrando la puerta a opciones re-mercantilizadoras, relacionadas con la participación de entidades privadas. La privatización de los servicios de empleo favorecería la marginalidad de los servicios públicos y, por tanto, una mayor fragmentación del mercado de trabajo: esta es una línea que, reiteran, no debe ser traspasada. En este sentido, hay una defensa del espacio público como lugar "legítimo" desde donde combatir el desempleo, si bien los criterios de legitimidad para este espacio público se remiten, en ocasiones, a términos procedentes del ámbito mercantil: eficiencia, rentabilidad, adecuados recursos humanos, etc. El problema para los sindicatos no se relaciona con la disyuntiva público-privado, sino con otra entre lo moderno y lo que ya no funciona. A través de esta última dicotomía se conceptualiza lo inadecuado de este tipo de intervención, evocando las disfunciones de una burocracia estereotipada (papeleo excesivo, lentitud, lejanía con los administrados, etc.).

"El SPE necesita un observatorio potente del mercado de trabajo, así como ha de someterse a un proceso de profesionalización y especialización. Los mismos técnicos de desempleo atienden todo tipo de ofertas, de modo que es imposible que haya un diálogo de verdadero entendimiento entre el demandante y el desempleado. Además, el servicio es obsoleto y rígido, lo cual es fácil de comprobar en el anquilosamiento de las categorías profesionales" (representante de UGT, nivel confederal).

Los medios de la intervención clásicos se consideran, por parte de todos los actores en las entrevistas, como desfasados, de modo que se apuesta por un mayor uso de la tecnología (versus "burocracia”) para hacer frente a la descompensación existente entre las "políticas/actuaciones" y la "realidad". El uso de las nuevas tecnologías en la administración por parte de los usuarios será un buen ejemplo de esta lucha contra la burocracia, facilitando la comunicación entre los participantes en las estructuras de gobernanza y los administrados: implica filtrar las demandas, dudas y 
sugerencias de estos últimos, permitiendo, de nuevo, aumentar la tan ansiada eficacia a la vez que se consigue una mayor cercanía al ciudadano.

"Reducimos la presencia física en la oficina y libera recursos para hacer cosas más interesantes... Este cajero (Autoservef) permite cambiar la programación de la formación. El cajero ha dado una opción al ciudadano le da muchas posibilidades”. (Jefe de servicio de gestión de la intermediación laboral SERVEF).

En general, los actores sociales coincidirán en una apelación a modos de gobierno o a estrategias de intervención diferenciadas y flexibles frente a un referente de intervención "burocratizada, centralizada y uniforme", criticado por todos ellos. No obstante, cada uno de ellos arguye razones de base distintas a la hora de elaborar su crítica a la burocracia y defender una reforma modernizadora que aporte eficacia. Así, los actores locales se apoyan en la referencia a la "cercanía”, lo más cercano es ser más ágil, flexible y mejor ajustado a la realidad; las ETTs se escudan en el "discurso experto" para deslegitimar la intervención pública y defienden la privatización como una descentralización eficaz; por su parte, los sindicatos parecen mostrar una posición de mayor ambivalencia ya que defienden lo público desde un discurso ético-moral y, sin embargo, en algunas ocasiones, achacan su ineficacia a un defecto de funcionamiento que se subsana a través de recetas propias del ámbito mercantil (principios de rentabilidad y eficiencia).

En cualquier caso, con independencia de las razones todos ellos apuestan por el cambio hacia un modelo de gestión que dará lugar a complejas y "modernas" interrelaciones entre ellos cuya forma dependerá del equilibrio de fuerzas entre todos los actores, y que generará un reparto de responsabilidades determinado. Todos los actores quieren comer del pastel de la modernización y encuentran en él una posibilidad de alcanzar mayor poder y representación a través de la negociación, la coordinación, etc., por lo que alabarán las bondades de la participación, como veremos a continuación.

\subsection{Participación y paternariado.}

La participación de diversos actores en el ejercicio del gobierno es una de las características más destacadas de los nuevos modelos de gobernanza, e implica el establecimiento de relaciones contractuales y de subordinación entre ellos. No obstante, el consenso sobre el modelo no significa que no existan tensiones en su definición. Como venimos señalando, cada uno de los actores va a connotar la noción de modernización con matices muy diferenciados: en parte por su particular posición en el proceso de intervención, pero también porque, además de dar significado a este proceso, están construyendo su identidad como entidad y están negociando su espacio simbólico en el "mercado de la inserción”. Por otro lado, hay un acuerdo en que la solución al problema del desempleo pasa por una acción negociada entre distintos actores sociales, siguiendo los principios de los modelos 
de partnership o partenariado (Stuart y Martínez Lucio, 2005). Se observa además una alusión a la búsqueda de estrategias de concertación y coordinación en los espacios de intervención, dentro de una lucha por la adquisición de competencias de gestión y, a la vez, con la idea de sumar esfuerzos. Dada esta multiplicación de actores, se critica la falta de un claro liderazgo, la fragmentación de lo público, su ausencia como "agente útil" en el nuevo panorama. Los actores sociales denuncian la escasa eficacia de las intervenciones de los servicios públicos, que más que aunar sus esfuerzos a fin de dar una respuesta adecuada a esta compleja situación les impelen a una situación de difícil competencia entre entidades. La fragmentación, más que colaborar al desarrollo de mecanismos para una adecuada inserción, parece dificultarla, perjudicando, en última instancia, al desempleado.

"no hay una buena coordinación entre las administraciones estatales y las autonómicas (...) Lo principal sería trabajar de forma coordinada, con una participación de todos los interlocutores” (representante de Cáritas España)”

"No se pueden atender políticas activas o fomento del empleo si no hay coordinación entre las tres administraciones” (Responsable técnico de la FEMP).

El requisito para que el modelo de partenariado funcione es la coordinación entre actores: a fin de favorecer una atención de calidad a la persona desempleada, han de comportarse como "colaboradores" (en lugar de como "contrarios" o "enfrentados"). Por tanto, se genera una retórica del consenso y del pacto, sostenida por todos los actores, y que lleva aparejado el reconocimiento de la necesidad de participación de diversos actores representantes de la sociedad civil, como ejemplo de una actuación responsable y moderna. La cuestión sería ahora hacia quién van dirigidas estas pretendidas acciones coordinadas, cuál sería el grupo referente para la recepción de estas políticas. La indefinición al respecto genera, a priori, una situación de competencia por los recursos. El espacio de la intervención se ha convertido en un lugar de pugna entre diversos actores sociales por acometer las labores que consideran de su atribución.

Los actores reclaman así, en las entrevistas, no sólo una participación en la implementación de las políticas sino también en su diseño, esto es, la participación en las distintas fases de la producción de la política (tanto en la definición de las categorías organizadoras del problema como en el reconocimiento de la autoridad política de sus representantes). Desde las ONG se fundamenta este "derecho a la participación” en su condición de ámbito natural para hacer frente al problema de la exclusión. En general, critican un sistema de intervención basado en subvenciones, estando más a favor de la negociación desde arriba que de la suscripción de contratos de servicios de implementación con la administración (hacer propuestas de políticas sociales y percibir subvenciones remiten a dinámicas contrapuestas entre sí). Mientras, los sindicatos tratan de legitimar su intervención desde aquello que consideran sus competencias: mencionan sus conocimientos especializados y experiencia, autoproclamándose en algunos de sus discursos como "los que entienden de empleo”. Conocimiento, habilidades técnicas y experiencia avalarán 
una intervención eficaz y de calidad. Los actores tratan siempre de hacer mención a su capacidad de gestión de programas, dentro de una óptica de partenariado y consenso con otros actores. Pero no todo es cultura de consenso, eficacia y participación. Hay unos límites claros que no se debe traspasar, y que tiene que ver con la externalización de ciertas actividades en manos privadas.

En contraposición, las ETT no sólo sostienen una legitimación de su actividad como "expertos en el mercado de trabajo" sino que además denuncian el problema de la rigidez legal como obstáculo a la libertad de elección. Se quejan de una situación de marginalidad que sufren especialmente en España, y que es considerada ilegítima desde instancias internacionales (citando a la Unión Europea). Como medio de superación de esa marginalidad, proponen formas contractuales de relación entre agentes de empleo basadas en "la libertad de elegir". A pesar de lo que podría parecer, en este clima dónde la participación y pugna por la intervención entre actores son los protagonistas, el Estado no queda completamente desbancado de su posición en calidad de actor. En su mayor parte, los actores (entre ellos, particularmente, los actores sindicales) no creen que el Estado deba desaparecer, sino que debe liderar el arbitraje de este conglomerado de actores y acciones.

"Los desempleados no son nuestros, sino del gobierno autonómico y estatal: colaboramos en su inserción porque estamos en la lucha contra el desempleo pero no es nuestra responsabilidad, que son los trabajadores.....Sí participamos en los Pactos Territoriales y en la formación y acciones de orientación”. (Representante de UGT de la Comunidad Valenciana)

En resumen, podemos decir que, de nuevo, aparece una defensa del espacio público como lugar "legítimo" desde donde combatir el desempleo; sin embargo, los modos de combatirlo están inspirados en lógicas del ámbito mercantil. Esta colonización de los discursos y argumentos del mundo empresarial explica que se construya y defienda una visión híbrida de lo público, en donde justicia social y eficacia técnica se presentan indisociables. Este es uno de los pilares desde el que se construye el argumento de la modernización, y en el que la imagen de una Europa capaz de conciliar eficacia en la lucha contra el desempleo y, a la vez, promoción de la justicia social, ha jugado un papel muy importante. Junto a este diagnóstico común, conviven, también en lo que se refiere al partenariado y la participación de los actores, distintas perspectivas y matices diferenciadores. Las ONGs fundamentan su legitimidad para la participación, en un escenario de actores interventores que se relacionan entre sí como interpares, en la especialización para hacer frente a la exclusión que acredita su bagaje; los sindicatos la fundamentan en sus competencias adoptando un discurso entre técnico y ético que, en todo caso, manifiesta oposición a la intervención privada; las ETTs refieren la calidad que como "expertos en el mercado de trabajo" ofrecen. A pesar de estas diferencias, a través de las que se tratan de reafirmar las identidades propias de cada actor, todos ellos presentan como último fundamento para el establecimiento de lógicas de gobernanza conforme al partenariado las recomendaciones de la Unión Europea. 


\subsection{Orientación y terapias individualizadas como estrategias realistas.}

Los discursos de los actores sociales coinciden en que, en la actualidad, el nuevo escenario requiere dejar atrás los modos de intervención tradicionales, ligados, como se señaló antes, a las denominadas políticas pasivas, a su vez, consistentes en la transferencia de prestaciones económicas (seguro de desempleo). Los distintos actores expresan su oposición a las políticas pasivas a través de una retórica activa, basada en tres ejes: la individualización de las intervenciones, la contractualización de estas (y por tanto el énfasis en la participación y en los deberes del desempleado) y la revalorización del trabajo como fuente de ciudadanía (Serrano Pascual, 2007). Lo moderno va a ser promover las políticas activas, cuya base es la idea de atención individualizada y a la carta. Por tanto, es necesario que haya un técnico especializado acompañando la vida "pasiva” del desempleado para que, por sí mismo, se active y sea capaz de entrar en el mercado de trabajo. El papel de los orientadores y mediadores se aproxima bastante al de un psicólogo con su paciente; los registros de sentido con los que se busca legitimar su participación en este mercado de la intervención son fundamentalmente de corte moral, como por ejemplo, la apelación a la dignidad humana, que se hace sinónimo de participación laboral. La dignidad humana se encuentra, así, en la actividad.

"Hay personas que nunca van a poder trabajar en el mercado normalizado por su situación personal pero que en un entorno de empleo protegido sí pueden hacer una aportación a la sociedad que es mucho más rentable que tenerlos pasivamente con unas rentas determinadas o incluso sin rentas (...) tienen dignidad como personas y a los que la sociedad tiene que ofrecer un espacio de dignidad". (Representante de Cáritas España).

La orientación de los usuarios se convierte en la punta de lanza de las políticas de empleo, cumpliendo un papel de intervención pública terapéutica para preparar las conductas de los individuos ante la crisis del trabajo ya comentada en otros trabajos (ver por ejemplo Serrano Pascual, Fernández Rodríguez y Artiaga Leiras, 2012). La función de los poderes públicos bajo esta perspectiva de “orientación” consistirá en facilitar que se descubra lo que aparece oculto, estimular que el sujeto se autoayude, se conozca y pueda desarrollar sus "oscurecidas" potencialidades. Participar más e involucrarse más es, asimismo, la cuestión esencial para las ETT, que consideran que pueden aportar mucho más que los servicios públicos de empleo. Desde su punto de vista, la falta de ofertas de trabajo de los servicios públicos de empleo (que sólo cuentan con desempleados registrados porque "se apuntan para conseguir la prestación") se debe no sólo a la falta de confianza de los empleadores en los desempleados, sino además a su escaso conocimiento del mercado real de trabajo. Los distintos actores van a tratar de suplir esas carencias de la administración pública con sus propias aportaciones, muy vinculadas a una cierta forma de entender las políticas activas. Los discursos de todos ellos están trufados de referencias a las virtudes de la orientación, de la flexibilidad, de la participación. 
"Si se le da la importancia que tiene que tener, y el mercado funciona como tiene que funcionar, la orientación no solamente es una herramienta para desempleados sino que permite que un trabajador pueda encontrar otro empleo o acceder a otra rama profesional. El orientador es un profesional que te puede asesorar para dirigir tu carrera profesional” (Responsable empleo UGT Valencia).

Por lo tanto, una cierta forma de combatir el desempleo fundamentada en las políticas pasivas ha finalizado, y "la realidad" está imponiendo un nuevo orden laboral en el que sólo las políticas activas pueden sostener una aproximación a la lucha contra el desempleo. La modernización se naturaliza y se presenta como un cambio irrefrenable del curso de las sociedades actuales. Incluso se utiliza el recurso de la personificación para mostrar que es el actual mercado de trabajo el que exige un cambio de paradigma y ante una alusión a la permanente crisis o estado de shock del mercado de trabajo español, se apela a la responsabilidad y el esfuerzo de las personas para garantizar el buen devenir de este nuevo modelo.

"Este cambio de paradigma se justifica desde el actual mercado de trabajo, que exige por parte del desempleado un esfuerzo para adaptarse a un contexto laboral en continuo cambio, donde es fundamental formarse permanentemente para adecuarse a las necesidades de este mercado y buscar activamente empleo" (Consejero Técnico de la Subdirección General de Políticas Activas del INEM).

Desde los diferentes Servicios Públicos de Empleo se van a hacer referencias continuas a la necesidad de hacer una apuesta por las políticas activas, las únicas políticas "morales" frente al conformismo y falta de compromiso que han generado las pasivas.

"El aumento del peso de las políticas activas de empleo sobre las pasivas... ha supuesto un acierto y se constituye en la base de toda política de empleo. La preparación del desempleado para poder desenvolverse en el mercado de forma autosuficiente es incuestionable...Las políticas pasivas están superadas, no hay lugar para el debate respecto a este punto, ambos interlocutores critican la implantación de determinados programas que suponen subsidios encubiertos; por definición, las políticas de empleo deben ser incentivadoras y no asistencialistas” (Área de fomento del empleo del SERVEF valenciano).

En este fragmento se percibe la rotundidad con que un nuevo tipo de seguridad activadora que permita "desenvolverse en el mercado de forma autosuficiente", la que entraña el concepto de flexiguridad europeo, es defendida por los propios servicios públicos. Se plantea, así, una dicotomía clave entre lo activo y lo pasivo. El impulso hacia la activación irá acompañado de una disminución en los esfuerzos dirigidos anteriormente a potenciar la protección social "pasiva", centrada en las prestaciones económicas para los desempleados. Así, se afirma que "Tenemos que pasar de yo te doy políticas, te doy cursos, yo te voy dando...sino que tú te tienes 
que ir buscando cuál es tu desarrollo profesional...” (Servicio de Orientación, SAE). No hay cortapisas a la hora de reconocer que hay una apuesta clara por la asunción de responsabilidades por parte del desempleado, al que se busca "incentivar". La prestación económica ya no significa una compensación por los abusos y la relación de poder que existe sobre los trabajadores en el mercado de trabajo. Ahora constituye únicamente un apoyo para poder efectuar las transiciones entre un trabajo y otro, en un contexto de exaltación de la flexibilidad laboral externa. Se está alerta ante cualquier conducta que pueda ser considerada como fraudulenta (te envían a gente que no quiere trabajar..., SERVEF valenciano; pensamos que todo el mundo es bueno, ¿eh? de entrada. Que todo el mundo quiere trabajar; SAE Andalucía), y por tanto, se asume que se debe restringir el derecho a recibir la prestación por desempleo con su correspondiente sanción.

Ahora, el seguro de desempleo pasa a considerarse como una herramienta disciplinadora más (Martín Martín, 2013), ya que sólo debe corresponder a aquellos que ofrezcan una buena conducta y que puedan demostrar que tienen una actitud de disposición a colaborar, de encontrar trabajo sea como sea. La percepción de rentas está mal considerada durante la búsqueda de empleo, al no proceder estas de la relación laboral. Se desplaza la representación de estas rentas hacia la de ayudas que desincentivan los esfuerzos para mejorar la empleabilidad y compromiso de activación de los parados, expresado este último en la asunción de mayor responsabilidad respecto a uno mismo en la trayectoria laboral y formativa. Además, se considera, por parte de la mayoría de los actores, que las políticas activas deberían ir dirigidas sólo a las personas que realmente están motivadas, ya que sino es una pérdida de tiempo y de recursos.

“...otros programas, son claramente asistencialistas y desincentivadores, como ocurre en el caso del pago al desempleado que participa en un IPI, no resuelven la situación del desempleado y premian económicamente su participación en acciones de empleabilidad que son de por sí, ya que se suscribe el compromiso de actividad, una obligación por definición...Colapsan la gestión y no son efectivas...” (SERVEF Valencia).

Este nuevo discurso que incide en la personalización de la intervención y el trabajo terapéutico con el desempleado se inserta en una lógica de incentivos y coacción, donde la vertiente coactiva aparece revestida de "compromiso" y "negociación", con un aparente interés en dotar de un rol participativo al desempleado (“colaborador”) en su relación con los programas de empleo. Este tipo de lógica promueve, en el fondo, un nuevo régimen disciplinario que fomenta el autodisciplinamiento, según el cual, es el propio individuo quien tiene que abrazar un proceso de autoayuda individual. A los sujetos se les reclama poner en juego su subjetividad y voluntad en el proceso de inserción. Estas nuevas demandas implican así la construcción social de nuevos sujetos-trabajadores no dependientes, que deben actuar con autonomía y una alta cuota de responsabilidad (Crespo Suárez y Serrano Pascual, 2007). Y sobre todo, asumir, por parte de la fuerza laboral, que el mundo ha cambiado y que ahora los trabajadores deben estar siempre preparados 
para un cambio, de carácter continuo, que les exige formarse y adaptarse a contextos caracterizados por la incertidumbre, la asunción de mayores riesgos, y nuevas oportunidades (Fernández Rodríguez, 2007). A este respecto, existe entre los distintos actores entrevistados una visión más o menos coincidente.

\section{Conclusiones. "Nos coordinamos entre nosotros": poder y responsabilidad en un contexto de cambio.}

Al analizar la lógica interna de las representaciones sobre la modernización se vislumbran distintas percepciones sobre cómo ha de ser entendida esta en relación a los servicios públicos de empleo, esto es, cómo se concibe su "deber ser". La referencia a la modernización entraña una transformación de los fundamentos políticos e ideológicos de la administración que, en parte, se construyen por oposición al modelo anteriormente vigente. En este proceso de "reinvención del gobierno" (Osborne y Gaebler, 1992) fundamentado en una serie de premisas básicas comunes destacadas a través de los ejes de análisis (sub-epígrafes de la sección tercera) pugnan fundamentalemente dos marcos discursivos que emanan de los discursos de diversos actores sociales: el enfoque neopúblico y la aproximación neoempresarial (Fernández Santos et al., 2008). El enfoque neopúblico parte de un principio categorial basado en el reforzamiento de los valores de lo público, y en un lenguaje ético, en donde los principios de universalidad y equidad son nucleares, y en donde se cuestiona que todo se pueda externalizar. Este discurso es, en buena medida, el de los actores sindicales que, no obstante, reconocen una imposibilidad de resistirse a la oleada contractualista y descentralizadora ("es imposible volver al inicio”) y reconocen en los métodos del partenariado y la coordinación alguna clase de mal menor ante la nueva realidad que se impone. Junto a los sindicatos, algunos organismos sin ánimo de lucro rescatan al Estado, desde su nueva posición de completa deslegitimación, como entidad de arbitraje en este clima de coordinación. Por otra parte, el discurso de otros actores, como los representantes de las ETT y los propios representantes de servicios públicos de empleo, es un discurso mucho más impregnado de un lenguaje privatizador, dirigido a reforzar la eficacia y eficiencia, y que adopta gran parte de los conceptos de la nueva cultura managerial presente en las empresas actuales (discurso "neoempresarial”).

Ambos discursos responden a la crisis de legitimidad ante la que se ve expuesto el Estado en las últimas tres décadas, pero cada uno de ellos enfatiza distintos conceptos: si el primero se ampara en una ética redistributiva, el segundo se inspira en un modelo de acción racional que aboga, como principio de comportamiento, el cálculo cuantificable de costes y beneficios. Si en el primer caso el sujeto de referencia es un ciudadano, en el segundo se trataría de un cliente-consumidor enfrentado ante un "mercado de la inserción", y preocupado por una asignación eficiente de los recursos y la maximización de la satisfacción del cliente. En este último caso, se plantea una necesaria reforma de los mecanismos (y argumentos) de mercado en el seno de la gestión pública. Muchas de las técnicas de gestión 
aplicadas en el ámbito empresarial, como son la demanda de flexibilidad, descentralización, evaluación, etc., son incorporadas en los discursos de los actores, participando así de una nueva "cultura de intervención".

En resumen, la descentralización y la participación múltiple, de un modo u otro, se presentan en los discursos de los actores sociales como realidad ineludible y situación de gobernanza irrevocable, con lo que el Estado se enfrenta, al final, a una tesitura de redefinición de su rol. La coordinación se torna la garantía de una atención adecuada al ciudadano, ya que "los instrumentos de coordinación" permiten "que la persona desempleada reciba esta oferta múltiple de forma ordenada y con sentido para su inserción laboral” (como señalaba un representante de CCOO a nivel confederal). La coordinación va a ser un potente referente legitimador de la gestión múltiple y multinivel: confiere a ésta el ideal de buena gestión al proporcionar coherencia a las intervenciones para/con el desempleado y genera alianza entre todos los actores neutralizando los conflictos de intereses. En la coordinación parece residir la esperanza del buen funcionamiento del nuevo sistema y su sola mención, como recurso político, es obligada y suficiente. El Estado ha de tener un rol más de árbitro que de actor participante. En cierto modo, el mensaje enviado desde los diferentes participantes en el proceso podría ser interpretado como el de un "tranquilos, ya nos coordinamos entre nosotros", apelando a la capacidad más que suficiente de los distintos actores para enfrentarse a la situación de desempleo, sin necesidad de engorrosos filtros y procesos burocráticos.

Concluiremos este trabajo señalando que la demanda de modernización de los servicios de empleo por parte de las instituciones europeas -con el fin de adaptarlos al nuevo paradigma de la activación y la flexiguridad- no ha caído en España, como hemos podido comprobar, en saco roto. Durante las dos últimas décadas se ha profundizado en un conjunto de reformas que han perseguido la adaptación del sistema de desempleo español a un marco basado en políticas activas, posteriormente absorbido por el de flexiguridad, si bien la cascada de cambios legislativos y reformas ha generado un modelo inestable, confuso y caracterizado por las contradicciones y los anacronismos. El resultado final parece que dista de satisfacer a los principales actores implicados en el desarrollo del mismo. El modelo se caracteriza, esencialmente, por una situación de confusión en la que el vacío de gobierno ha hecho presencia, al deslegitimarse fuertemente el modelo de intervención institucional sin haber asumido los actores participantes plenas responsabilidades para enfrentarse a un contexto en transformación. La modernización, además, se ha identificado con un rechazo a la burocracia pública, favoreciendo en su lugar intervenciones individualizadas y estrategias de concertación que, ante todo, implican una verdadera evacuación de lo social en las relaciones de empleo españolas.

Esto merece una detallada reflexión ética, política y sociológica, no solamente por cuanto supone la eliminación de unos elementos, los colectivos y sociales, que son la base de nuestro estatus como ciudadanos, sino porque esta visión de la modernización ha podido tener su influencia en el desarrollo de las políticas de empleo que se han comenzado a poner en marcha a partir del desplome de la 
actividad económica y el empleo (por ejemplo, con el reciente desmoronamiento de la coordinación ante la fiebre reformista gubernamental). El interrogante que se abre ahora es saber, en un contexto de crisis profunda como el actual, cómo van a impactar las presentes y futuras reformas de los servicios públicos de empleo, y si van a existir cambios profundos y/o posibles disensos en la concepción de dicha modernización. 


\section{Bibliografía.}

Alonso, L. E. (2007). La crisis de la ciudadanía laboral, Barcelona, Anthropos.

Alonso, L. E., Fernández Rodríguez, C. J. (2006). "El imaginario managerial: el discurso de la fluidez en la sociedad económica”. Política y Sociedad, 43 (2): $127-151$.

Alonso, L. E., Fernández Rodríguez, C. J. (Eds.) (2012). La financiarización de las relaciones salariales: una perspectiva internacional. Madrid: FUHEM / La Catarata.

Alonso, L. E., Fernández Rodríguez, C. J. (2013). Los discursos del presente: un análisis de los imaginarios sociales contemporáneos. Madrid: Siglo XXI.

Bonvin, J. M. (2004). "La rhétorique de l'activation et ses effets sur la définition des publics Cibles des politiques d'intégration sociale”, en Serrano Pascual, A. (Ed.), Activation polities for young people in an international perspective, Bruselas, ETUI : 101-129.

Crespo Suárez, E., Serrano Pascual, A. (2007). "Political production of individualised subject in the paradoxical discourse of the EU institutions", en van Berkel, R. y Vankelburg, B. (Eds.), Making it personal: Individualising Activation Services in the EU, Londres, Policy Press: 107 -126.

Du Gay, P. (2012). En elogio de la burocracia, Madrid, Siglo XXI.

European Commission (2007). "Towards common principles on flexicurity: More and better jobs through flexibility and security”, Employment and Social Affairs, Brussels, European Commission. Disponible en

http://ec.europa.eu/social/main.jsp?catId=117\&langId=en [acceso 12/04/2013].

Fairclough, N. (1995). Critical Discourse Analysis, Londres, Longman.

Fernández Rodríguez, C. J. (2007). El discurso del management: tiempo y narración, Madrid, CIS.

Fernández Santos, Y., Fernández Fernández, J.M., Rodríguez Pérez, A. (2008). "Modernización de la gestión pública. Necesidades, incidencias, límites y críticas”, Pecvnia, 6:75-105.

Kallenberg, A. (2009). "Precarious Work, Insecure Workers: Employment Relations in Transition”. American Sociological Review, 74 (1): 1-22.

Keune, M., Jepsen, M. (2007). "Not balanced and hardly new: the European Commision’s quest for flexicurity”, en Jørgensen, H. \& Kongshøj Madsen, P. (Eds.), Flexicurity and Beyond, Copenhague, DJØF Publishing.

Koch, M. (2006). Roads to Post-Fordism: Labour Market and Social Structures in Europe, Aldershot, Ashgate.

Kvale, S. (1996). Interviews: an Introduction to Qualitative Research Interviewing. Thousand Oaks, CA: Sage.

Landa Zapirain, J. P. (Ed.) (2009) Estudios sobre la estrategia europea de la flexiguridad: una aproximación crítica, Albacete, Bomarzo.

Laval, C., Dardot, P. (2013). La nueva razón del mundo. Ensayo sobre la sociedad neoliberal. Barcelona, Gedisa. 
Martín Artiles, A. (2002). “"Flexeguridad": Tiempo de trabajo y empleo en los pactos de empresa”. Sociología del Trabajo, 46: 71-96.

Martín Martín, M.P. (2013). Reinvención del gobierno del desempleo: Francia y España ante el marco referencial de la flexiguridad. Tesis doctoral. Universidad Complutense de Madrid, Madrid.

Martín Martín, M. P. (2009). "Questioning the employment system: the European flexicurity approach”. The journal of Industrial Relations and Human Resources, 11(5): 95-114.

Navarro, V. (2006). El subdesarrollo social de España: causas y consecuencias, Barcelona, Anagrama.

Osborne, D., Gaebler, T. (1992). Reinventing Government, Reading, Mass., Addison-Wesley.

Pérez Domínguez, F. (2007). "Servicios privados de empleo: razones para una reforma”. Documentación laboral, 79: 95-138.

Peters, B. G. (2005). "Gobernanza y burocracia pública: ¿nuevas formas de democracia o nuevas formas de control?”, Foro internacional, 182: 585-598.

Pollitt, C. (1993). El gerencialismo y los servicios públicos, Madrid, Instituto de Estudios Fiscales.

Pollitt, C. (2003). The essential public manager, Maidenhead, Open University Press.

Miguélez, F., Prieto, C. (2009). "Transformaciones en el empleo, flexibilidad y relaciones laborales en Europa”, Política y Sociedad, 46 (1-2): 275-287.

Santos Ortega, A., Martín Martín, P. (2012). "La juventud española en tiempos de crisis. Paro, vidas precarias y acción colectiva”. Sociología del Trabajo, 75: 93110.

Serrano Pascual, A. (2007). "Reshaping Welfare States: Activation Regimes in Europe”, en Serrano Pascual, A. y Magnusson, L. (Eds.), Reshaping Welfare States and Activation Regimes in Europe, Bruselas, P.I.E. Peter Lang: 11-35.

Serrano Pascual, A. (2009a).“The battle of ideas in the European field: The combat to defeat unemployment and the struggle to give it a name”. Transfer, 15 (1): 5371.

Serrano Pascual, A. (2009b). "Regulación supranacional y despolitización del trabajo: el caso del paradigma de la activación”, en Crespo, E., Prieto, C. y Serrano, A. (Dirs.), Trabajo, subjetividad y ciudadanía. Paradojas del empleo en una sociedad en transformación, Madrid, Editorial Complutense / CIS: 259290.

Serrano Pascual, A., Fernández Rodríguez, C. J., Artiaga Leiras, A. (2012). "Ingenierías de la subjetividad: el caso de la orientación para el empleo", Revista Española de Investigaciones Sociológicas, 138: 41-62. DOI:10.5477/cis/reis.138.41

Serrano Pascual, A. Magnusson, L. (Eds.) (2007). Reshaping Welfare States and Activation Regimes in Europe, Bruselas, P.I.E. Peter Lang.

Silverman, D. (1993). Interpreting Qualitative Data. Methods for Analysing Talk, Text and Interaction. Londres, Sage. 
Sobrino González, G. (2007). La política de colocación, Albacete, Bomarzo.

Sol, E., Westerveld, M. (Eds.) (2005). Contractualism in Employment Services. A new reform of welfare state governance, La Haya, Llumer Law International.

Stuart, M., Martínez Lucio, M. (Eds.) (2005) Partnership and Modernisation in Employment Relations, Londres, Routledge.

Tovar, F.J. y Revilla, J.C. (2012) La institucionalización de la individualización del trabajo: el concepto de flexiguridad. Cuadernos de Relaciones Laborales, 30 (1): 235-258.

Wilthagen T., Tros, T. F. (2004). "The Concept of Flexicurity: A New Approach to Regulating Employment and Labour Markets”. Transfer, 10 (2): 167-186.

Wodak, R., Meyer, M. (Eds.) (2009). Métodos de análisis crítico del discurso, Barcelona, Gedisa.

Valdés Dal-Ré, F. (2006). "Intermediación laboral: apuntes críticos de una reforma socialmente visible y normativamente provisional”. Relaciones laborales, 19, editorial.

Vallés, M. (2002). Entrevistas cualitativas. Madrid: CIS. 
ANEXO: Datos de las entrevistas.

\begin{tabular}{|l|c|}
\hline \multicolumn{1}{|c|}{ Perfil de Entrevistados } & Entrevistas \\
\hline Representante de UGT a nivel nacional & 1 entrevista \\
\hline Representante de CCOO a nivel nacional & 1 entrevista \\
\hline Representante de UGT en la Comunidad Valenciana & 1 entrevista \\
\hline Representante de CCOO en la Comunidad de Andalucía & 1 entrevista \\
\hline $\begin{array}{l}\text { Representante de Federación Española de Municipios y } \\
\text { Provincias }\end{array}$ & 1 entrevista \\
\hline $\begin{array}{l}\text { Representante de Federación Valenciana de Municipios y } \\
\text { Provincias }\end{array}$ & 1 entrevista \\
\hline $\begin{array}{l}\text { Representante de Federación Andaluza de Municipios y } \\
\text { Provincias }\end{array}$ & 2 entrevistas \\
\hline Responsable políticas activas - INEM & 1 entrevista \\
\hline $\begin{array}{l}\text { Responsable de políticas activas Servicio Andaluz de } \\
\text { Empleo }\end{array}$ & 3 entrevistas \\
\hline $\begin{array}{l}\text { Responsable de políticas activas Servicio Valenciano de } \\
\text { Empleo y Formación }\end{array}$ & 4 entrevistas \\
\hline Representante de Cáritas a nivel nacional & 1 entrevista \\
\hline $\begin{array}{l}\text { Representante de Asociación de Grandes Empresas de } \\
\text { Trabajo Temporal a nivel nacional }\end{array}$ & 2 entrevistas \\
\hline & $\mathbf{1 9}$ entrevistas \\
\hline
\end{tabular}

